\title{
Broadband extreme ultraviolet interferometry and imaging
}

\author{
Matthijs Jansen ${ }^{1,2}$, Anne de Beurs ${ }^{1,2}$, Kevin Liu ${ }^{1,2}$, Kjeld Eikema ${ }^{1,2}$ and Stefan Witte ${ }^{1,2, *}$ \\ ${ }^{1}$ Advanced Research Center for Nanolithography, Science Park 110, 1098 XG Amsterdam, The \\ Netherlands \\ ${ }^{2}$ Department of Physics and Astronomy, and Laserlab, Vrije Universiteit, De Boelelaan $1081 \mathrm{HV}$ \\ Amsterdam, The Netherlands
}

\begin{abstract}
Using a pair of phase-locked high-harmonic generation sources, we demonstrate Fourier transform interferometry at extreme-ultraviolet (EUV) wavelengths between 17 and $55 \mathrm{~nm}$. This is made possible by the adaptation of a birefringence-based ultrastable interferometer for infrared femtosecond pulses. Since we measure the interference with an EUVsensitive CCD camera, this enables a wide range of spatially and spectrally resolved measurements at extreme ultraviolet wavelengths. We demonstrate the capabilities of this technique by performing wavelengthresolved high-resolution coherent diffractive imaging and by measuring the spatially resolved spectral absorption of a thin structured titanium film.
\end{abstract}

\section{Interferometry}

High-harmonic generation (HHG) is a well-known source of broad extreme ultraviolet (EUV) and soft-X-ray spectra. Due to the excellent spatial coherence of the generated light, HHG-sources are an ideal candidate for interferometry at these wavelengths. It has already been shown that high-harmonic interferometry is a useful tool to determine various source properties, such as spectra and wavefronts of the individual high-harmonics. But the low temporal coherence of these ultrabroadband HHG sources does require dedicated methods to perform interferometry, and a particularly interesting approach is based on Fourier transform spectroscopy (FTS). In FTS, a measurement of the temporal interference of a pair of identical pulses provides an autocorrelation of the pulse. A numerical Fourier transform then yields an intensity spectrum of the light.

FTS is a widely used technique for visible and infrared wavelengths, but application to EUV wavelengths has been limited due to several experimental difficulties. First, FTS requires control and stability of the pulse delay at a sub-wavelength level. For extreme ultraviolet wavelengths, this translates to attosecond (or nanometer) control of the pulse delay, which is difficult to obtain using standard interferometers. Furthermore, the generation of pulse pairs is hindered by the lack of EUV beamsplitters.

We demonstrate that these challenges can be overcome using a common-path interferometer based on birefringent wedges to generate a pair of infrared pulses with which

\footnotetext{
*Corresponding author: s.witte@arcnl.nl
} 
the HHG can be driven [1,2]. A piezo-driven translation stage is used to position one of the wedges, controlling the pulse delay at the attosecond level. The common-path interferometer is suitable for the 5 millijoule, 25 femtosecond pulses generated by our noncollinear optical parametric chirped pulse amplifier, and a slight tilt of one of the wedges introduces a small angle between the pulses. This separates the IR pulses spatially in the high-harmonic generation region, enabling the generation of phase-locked EUV pulse pairs with a controllable delay and a smooth spatial intensity profile, in both argon and neon gas. The setup and a typical EUV beam profile including interference of the two pulses for HHG in argon are shown in Figs. 1a and 1b. Figure 1c shows a typical time delay scan recorded on a single camera pixel for the case of HHG in neon. The observed signal is the temporal coherence function of the HHG attosecond pulse train, with individual pulses having a coherence time of 120 attoseconds (Fig. 1d). A detailed analysis of the recorded interference scans shows that the common-path interferometer achieves an RMS timing stability of 0.8 as. Taking the Fourier transform of the time delay scan yields the HHG spectrum (Fig. 1e), spanning the wavelength range of 17 to $55 \mathrm{~nm}$.

(a)
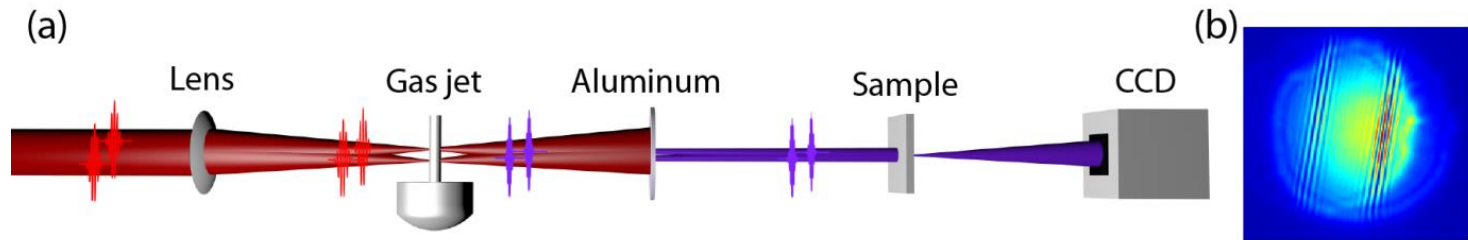

(c)

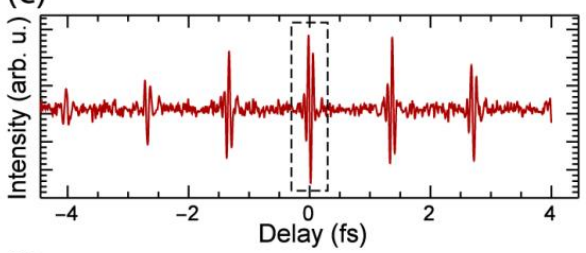

(f)

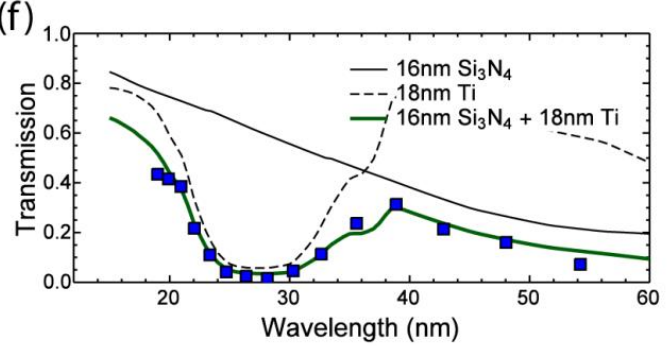

(d)

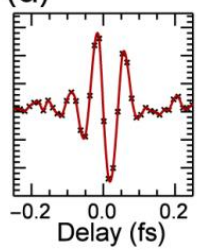

(g)

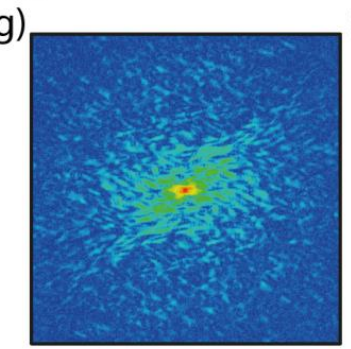

(e)

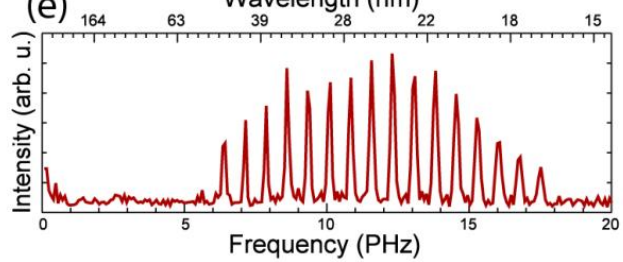

(h)

Fig. 1. Spatially-resolved Fourier-transform spectroscopy of high-harmonics. (a) Schematic overview of the high-harmonic generation setup. The output of the interferometer is focused in a gas jet to generate a pair of EUV pulses. A thin Aluminium filter is used to separate the EUV from the infrared, after which a transmissive sample can be positioned in the beam using a remotely controlled translation stage. An EUV-sensitive camera is used to detect the transmitted light. (b) Observed interference of the two EUV pulses in the absence of a sample. (c) Single pixel interference trace recorded while generating high-harmonics in neon and without a sample in the beam. (d) Zoom in of the centre portion of (c). (e) Spectrum of the high-harmonics generated in neon, averaged over ten neighbouring pixels. (f) Spatially resolved FTS measurement of the transmission of a thin Ti/Si3N4 film, compared to a reference spectrum transmitted through a hole in the same film. The spectral transmission matches with the expectations and correctly retrieves the thicknesses of the Titanium and Silicon Nitride. (g) Monochromatic diffraction pattern of a small isolated sample at $37 \mathrm{~nm}$ wavelength, measured using spatially resolved Fourier-transform spectroscopy with broadband highharmonics. (h) Numerical reconstruction of the sample from the diffraction pattern in (g). 


\section{Spectrally resolved imaging}

Using an EUV-sensitive CCD camera to detect spatially resolved interference and diffraction patterns, our EUV interferometry approach enables spatially resolved Fouriertransform spectroscopy of high-harmonics. This technique can be used to measure the spectral absorption of complex samples. We demonstrate such a measurement using a 20 $\mathrm{nm}$ titanium film on a $15 \mathrm{~nm}$ silicon nitride membrane, in which we drilled a 100 micron diameter hole using a focused ion beam. While it would be difficult to measure the spectral transmission of the hole and film separately using a grating spectrometer, this measurement is readily performed using spatially resolved FTS. Illuminating the sample with highharmonics generated in neon, we were able to directly measure the absorption of the thin film in the wavelength range of 17-55 nm (see Fig. 1g) [2].

Extending to higher spatial resolution is possible by combining spatially resolved FTS with coherent diffractive imaging (CDI). In CDI, an image of the sample is reconstructed based the measurement of a far field diffraction pattern. As the resolution of the reconstructed image is not limited by optics, CDI is a widely used technique for EUV and $\mathrm{X}$-ray microscopy. Normally, coherent and monochromatic illumination is necessary in order to get good diffraction patterns. Although HHG provides a table-top coherent source of EUV radiation, the use of HHG for coherent diffractive imaging has been limited because of the need for spectral filtering. Using spatially resolved FTS of high-harmonics, it becomes possible to record monochromatic diffraction patterns for all wavelengths in the illumination simultaneously, enabling efficient spectroscopic imaging using the full bandwidth produced in high-harmonic generation. An example of such a diffraction pattern is shown in Fig. 1g. Due to the noncollinear two-beam geometry of this experiment, the recorded diffraction pattern differs slightly from the traditional far-field diffraction pattern. Therefore, a custom phase retrieval algorithm has been used to reconstruct the image shown in Fig.1h [3].

\section{References}

1. D. Brida, C. Manzoni, G. Cerullo, Opt. Letters, 37(15), 3027-3029 (2012).

2. G.S.M. Jansen, D. Rudolf, L. Freisem, K.S.E. Eikema, S. Witte, Optica 3(10), 11221125 (2016).

3. G.S.M. Jansen, A.C.C. de Beurs, X. Liu, K.S.E. Eikema, S. Witte, Opt. Express 26(10), 12479-12489 (2018) 